\title{
Erratum to: Evolution of Black Holes in Anti-de Sitter Spacetime and the Firewall Controversy
}

Yen Chin Ong

Erratum to:

Y.C. Ong, Evolution of Black Holes in Anti-de Sitter Spacetime and the Firewall Controversy, Springer Theses, DOI 10.1007/978-3-662-48270-4_7

In the original version of the book, the last paragraph in p. 56, which continues in p. 57, in Chap. 2 and the sentence below the Eq. B.31 in Backmatter have to be replaced with the new contents. The erratum book has been updated with the changes. 